\title{
SPURIOUS BROWNIAN MOTIONS ${ }^{1}$
}

\author{
SÁNDOR CSÖRGO
}

ABSTRACT. Spurious Brownian motions are characterized in $\mathbf{R}^{d}, d \geqslant 2$.

Let $W=\left\{W(t)=\left(W_{1}(t), \ldots, W_{d}(t)\right), t \geqslant 0\right\}$ be an $\mathbf{R}^{d}$-valued mean-zero Gaussian process such that all projections $Y_{\lambda}(t)=\sum_{j=1}^{d} \lambda_{j} W_{j}(t)$ behave as if $W$ were standard Brownian motion in $\mathbf{R}^{d}$, i.e., $E Y_{\lambda}(s) Y_{\lambda}(t)=\min (s, t) \sum_{j=1}^{d} \lambda_{j}^{2}$. Following Hardin [1], we call $W$ a spurious Brownian motion if it is not a standard Brownian motion in $\mathbf{R}^{d}$. He showed by an example that such a process exists in $\mathbf{R}^{2}$.

Let $S(s, t)=\left(\sigma_{j k}(s, t)\right)$ denote the covariance matrix function of $W: \sigma_{j k}(s, t)=$ $E W_{j}(s) W_{k}(t), j, k=1, \ldots, d$. Necessarily, $\sigma_{j j}(s, t)=\min (s, t)$ for each $j=$ $1, \ldots, d$. Hence the identity $E Y_{\lambda}(s) Y_{\lambda}(t)=\sum_{j, k=1}^{d} \lambda_{j} \lambda_{k} \sigma_{j k}(s, t) \equiv \min (s, t) \sum_{j=1}^{d} \lambda_{j}^{2}$ is equivalent to the identity

$$
\sum_{j, k=1: j \neq k}^{d} \lambda_{j} \lambda_{k} \sigma_{j k}(s, t) \equiv 0 .
$$

This holds for any vector $\lambda=\left(\lambda_{1}, \ldots, \lambda_{d}\right)$ if and only if $\sigma_{j k}(s, t) \equiv-\sigma_{k j}(s, t)$ for all $j, k=1, \ldots, d ; j \neq k$. Thus $W$ is a spurious Brownian motion if and only if $S(s, t)$ is skew-symmetric and not diagonal.

Hardin's example in $\mathbf{R}^{2}$ is an example for such a covariance matrix with $\sigma_{12}(s, t)$ $=3^{-1}\{\min (2 s, t)-\min (s, 2 t)\}$. However, for any choice of the functions $\sigma_{j k}(s, t)$, $k=j+1, \ldots, d ; j=1, \ldots, d$, such that $\left|\sigma_{j k}(s, t)\right| \leqslant(s t)^{1 / 2}, \sigma_{j k}(s, t)+\sigma_{j k}(t, s) \equiv 0$, and that $\sigma_{j k}(s, t) \not \equiv 0$ for at least one pair $(j, k)$, there is a spurious Brownian motion in $\mathbf{R}^{d}$, provided that for any integer $m \geqslant 2$, any $t_{1}, \ldots, t_{m} \geqslant 0$, and any real numbers $\lambda_{i l}, i=1, \ldots, d ; l=1, \ldots, m$, we have

$$
\sum_{i, l=1}^{m}\left\{\min \left(t_{i}, t_{l}\right) \sum_{j=1}^{d} \lambda_{j i} \lambda_{j l}+\sum_{j=1}^{d-1} \sum_{k=j+1}^{d} \sigma_{j k}\left(t_{i}, t_{l}\right)\left(\lambda_{j i} \lambda_{k l}-\lambda_{j l} \lambda_{k i}\right)\right\} \geqslant 0 .
$$

These conditions are necessary and sufficient for the existence of a mean-zero stochastic process $X=\left\{X(t)=\left(X_{1}(t), \ldots, X_{d}(t)\right), t \geqslant 0\right\}$ that has covariance matrix function defined as the skew-symmetric matrix corresponding to the functions $\sigma_{j k}(s, t), k=j+1, \ldots, d ; j=1, \ldots, d$, and having diagonal $\sigma_{j j}(s, t)=\min (s, t)$,

Received by the editors May 3, 1985 and, in revised form, August 15, 1985.

${ }^{1}$ Work done while the author was a Visiting Professor at the Department of Mathematics, University of California at San Diego and at the Department of Statistics, Stanford University.

1980 Mathematics Subject Classification. Primary 60G15, $60 \mathrm{~J} 75$.

Kel words and phrases. Brownian motion, projection. 
$j=1, \ldots, d$. The projections of $X$ already behave as if $X$ were a standard Brownian motion in $\mathbf{R}^{d}$, though $X$ is not necessarily Gaussian. If $X$ is Gaussian, and such an $X$ exists, then it is necessarily a spurious Brownian motion in $\mathbf{R}^{d}$.

\section{REFERENCES}

1. C. D. Hardin, Jr., A spurious Brownian motion, Proc. Amer. Math. Soc. 93 (1985), 350.

BOI.yai Institute, SZEgEd University, 6720 SZEgED, HUNGARY 